\title{
MODELLING INCIDENT SCENARIOS
}

To enrich User Interface Development

Claudia V. S. Guerrero ${ }^{1}$, Maria de F. Q. V. Turnell ${ }^{1}$, Jean-Marc Mercantini' ${ }^{2}$, Eugène Chouraqui ${ }^{2}$, Fernando A. Q. Vieira ${ }^{3}$ and Madson R. B. Pereira ${ }^{4}$

${ }^{1}$ Laboratório de Interfaces Homem Máquina da Universidade Federal de Campina Grande Caixa Postal 10053 CEP 58109-970 Campina Grande PB - Brazil; ${ }^{2}$ Laboratoire des Sciences de l'Information et des Systèmes, de l'Universitè d'Aix-Marseille III, Domaine Universitaire de Saint-Jerôme, Avenue Escadrille Normandie-Niemen 13397 Marseille Cedex 20-France; ${ }^{3}$ DOMO-DOS-SOC-DO and ${ }^{4} S L O G-D O$ at CHESF - Companhia Hidro Eletrica do Sao Francisco, Rua Delmiro Gouveia, 333 - Bongi, CEP 50761-901, Recife PE, Brazil.

Abstract: This paper presents the process employed in obtaining a conceptual model of human errors scenarios for the electrical power industry. The model presented results from the analysis of the industry's reports on human and operational errors using a knowledge acquisition method (KOD). These scenarios will be used to build a process control simulator with which it will be possible to study the user behaviour when dealing with safety critical situations. From these studies it will be extracted a cognitive model of the user behaviour when working under critical situations to be incorporated into a method for the conception of user interfaces based upon ergonomic concerns (MCIE).

Key words: User interface Design, Process control, Human Error analysis, knowledge acquisition

\section{INTRODUCTION}

As result of technology development, industrial machinery and tools have reached a high degree of perfection in their performance transferring to the human operators the responsibility of almost all of the failures that happen during the interaction between them. According to Amalberti ${ }^{1}$ human errors have almost always been considered the main cause of accidents. This is the result of differences in work pace and in representation languages which lead into misunderstandings, responsible for the majority of 
accidents reported ${ }^{2}$. On the other hand, when attempting to manage their own abilities and error rate, the operators risk to increase their fatigue and stress.

This problem reaches higher proportions in industrial applications supported by complex systems considered safety critical from the viewpoint of the consequences of errors and faults, whether in financial terms or in terms of their catastrophic consequences. For those systems, beyond precision and functionality, it is imperative to offer their users: built in safety, adaptability to different degrees of expertise and work situations and support to easy the learning.

According to Amalberti ${ }^{1}$ the causes of human errors can be classified into two categories: (i) internal causes such as stress, fatigue, high cognitive loads associated to time pressure or lack of knowledge about the task and/or the system; and (ii) external causes such as badly conceived aids and problematic systems. A badly designed user interface may lead the operator into misinterpretations, causing decision making errors and putting at risk the system's operation. For these reasons and in these contexts, user interface adequacy becomes even more important and critical, since it is possible through its design to easy task completion and to reduce the cognitive loads.

The user always makes mistakes; it is necessary to accept them and try to ensure that they will not lead into accidents. The operator's risk perception causes an increase in safety margins when planning activities, an increase in the expected performance levels thus interfering with the way of thinking and decision making ${ }^{3}$. From the designer's point of view, it means to build systems and user interfaces more robust from the viewpoint of error tolerance, acknowledging the user's right to make mistakes. So, it is essential to increase error visibility in order to facilitate its correction. In spite of the designer's efforts, in offering manuals, help assistants, and other kinds of help, errors will always occur. So it is important to research help strategies which are closer to the user's mental representation and language ${ }^{1}$.

Difficulties in the communication between the designer and the final user have lead to considerable distances between the understanding of the task which the user actually performs and that which the designer assumes that the user must perform ${ }^{3}$. This is a vicious cycle which can only be broken by the ergonomical analysis of the user activity, by the positive feedback, and by the ergonomical validation of the tools developed to support the user activity. This ergonomical view of user interface design can be achieved by means of a user centred approach to interface design, which is based on the task analysis and on prototyping building and validation. 
This paper presents how to obtain a conceptual model for human errors scenarios, for safety critical systems in the electric power industry, using the method $\mathrm{KOD}^{4}$. This work is part of a joint research project supported by CAPES-COFECUB, which involves the LIHM-UFCG in Brazil, the LSISUniversite d'Aix-Marseille III, in France and the Brazilian Electric Power Company, CHESF.

The scenario model to be presented is the result of a preliminary study on the industry's database of incident and accident reports. This model will be used as the basis for building a process control simulator, with which it will be possible to study the user behaviour when dealing with safety critical situations. This approach is in accordance with Hollnagel's point of view which prescribes to consider the working context (critical situations) when modelling the user activity ${ }^{5}$. In these studies the user will be confronted with critical and atypical situations and his behaviour will be registered and analysed in order to build a user behaviour cognitive model when dealing with these situations. The observations will take place in the LIHM, with an infrastructure adequate for registering in different media the interactions between operator and simulator. From these studies it will be extracted a cognitive model of the user behaviour when working under critical situations to be incorporated into the MCIE Method for the Conception of Ergonomical Interfaces (Método para a Concepção de Interfaces Ergonômicas) ${ }^{6}$, which is based upon ergonomic principles.

This paper is organised as follows. Section 2 gives an overview of the methods and tools employed in this research. Section 3 introduces the case study and the criteria adopted to choose the corpus of reports from the industry's database. Section 4 describes how the incident scenario model was built using the method $\mathrm{KOD}^{4}$. Section 5 discusses the results of the study and presents directions for future work.

\section{FORMALISMS AND TOOLS}

When developing safety critical systems in particular, the Ergonomics focus lies on the user interface design. In this context, optimizing the cooperation between the user and the system to perform a task implies in reducing the semantical difference between the user's real world and the application's abstract world which not necessarily share the same working $\operatorname{logic}^{6}$. In the attempt to produce better user interfaces various methods propose to integrate the ergonomical knowledge earlier in the process. Amongst the methods which are based on task models are: ERGO-START ${ }^{7}$, $\mathrm{MACIA}^{8}, \mathrm{ALACIE}^{9}, \mathrm{MEDITE}^{10}$ and $\mathrm{MCIE}^{6}$. 
The conception of ergonomic human interfaces for industrial applications is the focus of the research at the Human Interface Group (GIHM) at UFCG. The method employed is the MCIE. This method has already been applied to the development and usability evaluation of industrial user interfaces ${ }^{11,12,6}$. At the centre of this group's research is the project which aims to extend the MCIE and its supporting environment to enable designers of interfaces for safety critical applications to be able to conceive and evaluate the design's ergonomical adequacy.

Each of the MCIE phases is supported by model building and the design process is centred on evaluation. Thus the result of each conception phase must be evaluated before proceeding to the following one. It adopts a user centred approach in order to achieve an ergonomic result adequate to the expectations and abilities of the users when performing their tasks under critical situations. However, the user cognitive behaviour is still to be explored by the current development methods which tend to concentrate on the information related to ergonomic work analysis such as age, sex, knowledge, background, work strategies, etc. It is also necessary to consider those related to the cognitive abilities, more specifically to understand and consider the user behaviour when facing risk and critical situations.

The MCIE research project aims to incorporate such knowledge into the UI interface design process by means of incorporating a model of the cognitive user behaviour into the requirement phase. The knowledge to be considered is specific to the context of operating industrial systems under critical situations. With this knowledge added to the requirements it is expected to arrive to more ergonomically adapted user interfaces thus reducing and even eliminating a great deal of incidents in the industry. In figure 1 it is presented the MCIE process, its phases, related models and tools, highlighting the introduction of the cognitive model.

The conceptual model of error scenarios presented in this paper was built using the method $\mathrm{KOD}^{4}$. This method uses a bottom up approach in order to maximise the data extraction both from the specialist and text documents, reordering those under a model of homogenous structure. The choice of the method KOD is due to the following features:

- Based upon linguistic engineering, well adapted to extract knowledge from text expressed in natural language (such as the incident reports).

- Follows a bottom up approach so the model is gradually constructed from the raw data and knowledge.

- Guides the engineer from the knowledge extraction to the software model.

KOD requires elaborating three successive models: the practical model, the cognitive model and the software model. Each one is based on the paradigm 
$<$ representation, action, interpretation $>$. A system development process using KOD results in the models represented in figure 2.

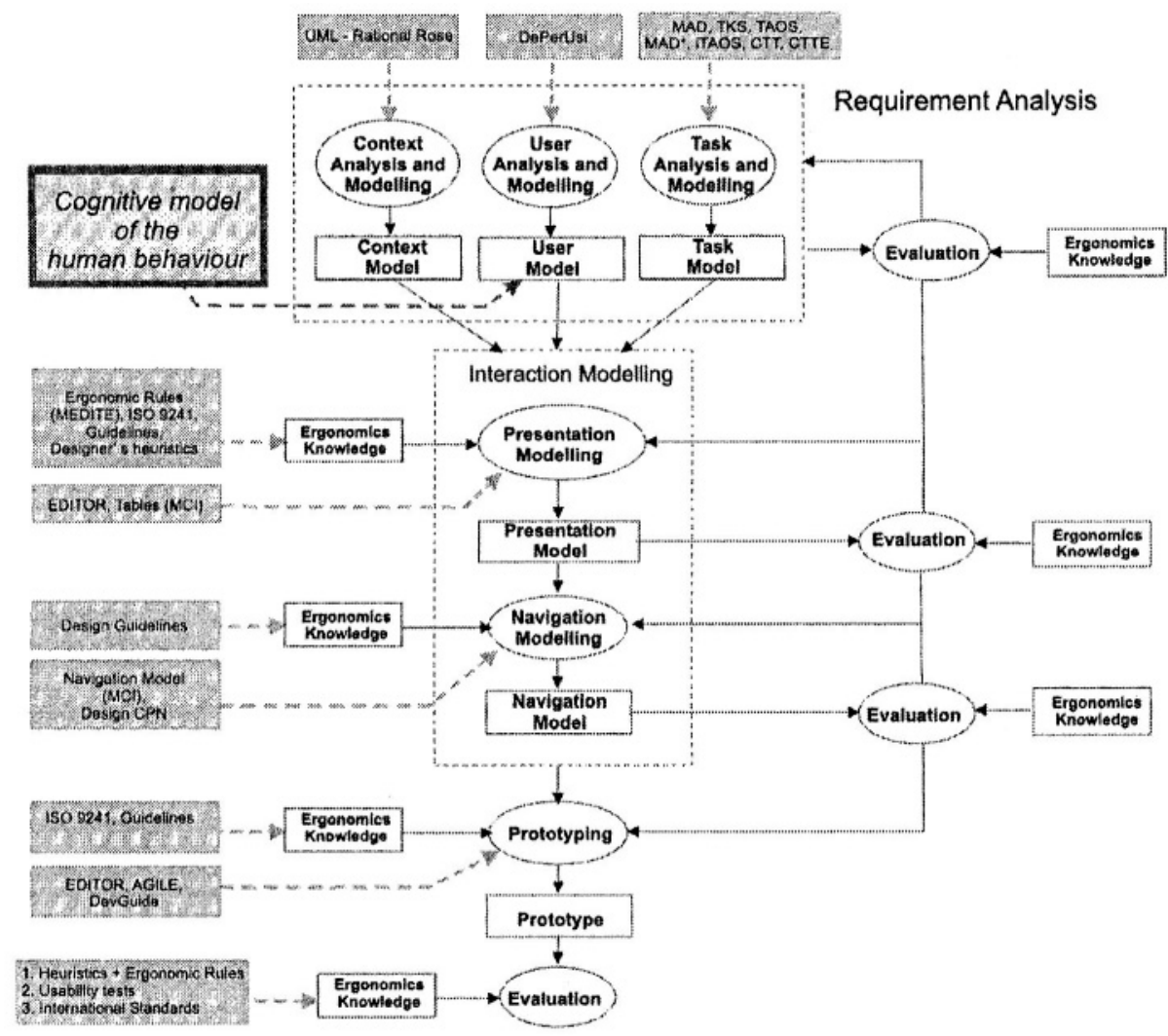

Figure 1, MCIE and the cognitive model of the user behaviour

This application of this method consists on the following steps:

- From each incident report a practical model (P.M) is constructed. This is the knowledge extraction operation.

- Based on the practical models, a cognitive model is elaborated. This is the abstraction operation.

- The software model is obtained through to the use of a formal language. This is the formalisation operation.

The KOD method constitutes a powerful framework to structure the domain knowledge. This method has already been applied to the domains: traffic accident modelling ${ }^{13}$ and urban industrial site simulation ${ }^{14}$. 


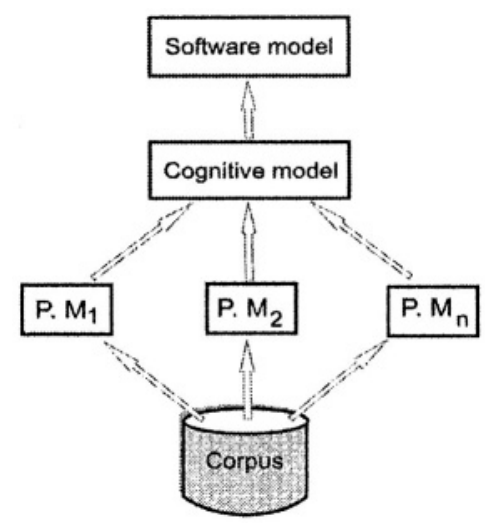

Figure 2. KOD Models

\section{CASE STUDY}

In the context of safety critical systems, a case study was chosen related to the electric power industry, in particular to the managing of the process involved in the transmission and distribution of electric power.

In the electrical Power Industry, according to annual reports on failures, a significant proportion of the incidents are due to human error. This industry is particularly interesting as a case study, not only because of its safety critical characteristics, but also because of its systematic approach in analysing and documenting failures. Also, it is an industry which mixes a high degree of automation in some of its operation centres but also keeps a good share of decision making and task performance on the hands of the operators. There, human errors may bring internal consequences such as material losses and incidents which may even endanger the lives of those who work for the industry, as well as the interruption of the service known as power supply cuts with the well known consequences to other industrial clients and to the society in general.

Typically, this process happens in a network of substations hierarchically organised into geographical regions, each of which associated to a control centre. The control centres manage the flow of electricity by supervising it and occasionally controlling specific substations, either remotely or via coordinated actions with the substation operators. Each substation has a set of input lines which brings in the electric power from the distribution network, which is then processed and passed on as outputs into the distribution network. The process may consist in changing the levels of electric tension or simply switching it between nodes of the network. The electric power 
supplied in the output lines of the substation can feed other nodes in the network or be directed to consumers of the industry.

With the technology development, in the higher levels of automation, the task performed by the operators of the supervisory and control systems in this industry has increased in complexity. During system operation the demand for almost immediate responses and fast decision making, with little or no tolerance to errors, leads to an increasing cognitive load.

In spite of all efforts, errors will always occur during the operation of such systems. In this study, we concentrated our efforts on incident reports due to human errors. The aim is to understand the cognitive behaviour during task execution under critical situations and thus develop human interfaces which account for cognitive ergonomics thus leading into higher levels of safety for both the operators and the system.

\subsection{Defining the Corpus}

Due to safety regulations the power supply industry keeps a detailed record of incidents and accidents along many years of operation. Initially, as a part of a preliminary study, 21 reports were chosen from the industry's database. These reports are related to different scenarios of incidents which happened in different locations in the industry's network of substations, in the past two years of operation. This period was chosen to ensure updated information in respect to work patterns and tasks. From the set of 21 reports it was extracted the corpus which constituted this preliminary study. In this section we synthesise the results of the corpus analysis.

The typical scenarios of incidents were related to system operation during the so-called manoeuvres which are classified in this industry as: routine, maintenance and emergency. From the preliminary study it was found that the causes associated to human errors can be classified as: (i) internal, such as stress, fatigue, and lack of attention and excess selfconfidence; and (ii) external such as insufficient documentation, faulty equipment and inadequate work conditions such as insufficient lighting.

A set of criteria was then adopted in order to select the documents relevant to the purposes of this study. The reports of interest for this preliminary study were about human errors related to tasks of control and supervision, performed either in the control room or in the patio of equipments. These criteria emerged from a first analysis resulting in an abstract written for each report. The next step consisted in synthesising all the relevant information on a table format highlighting: the task performed, the cause of the error (according to the point of view of industry's experts), the consequences of the error and a brief description of the scenario before and during the task execution which lead into error. This analysis made 
possible to identify the corpus relevant to this preliminary study. The corpus of this study consists of 8 reports on human errors and equipment failures which occurred on 8 different sites of the industry.

\section{BUILDING THE CONCEPTUAL MODEL FOR THE HUMAN ERROR SCENARIO}

In this section we introduce the process used in order to obtain the conceptual model of human errors using the method $\mathrm{KOD}^{4}$ (figure 3).

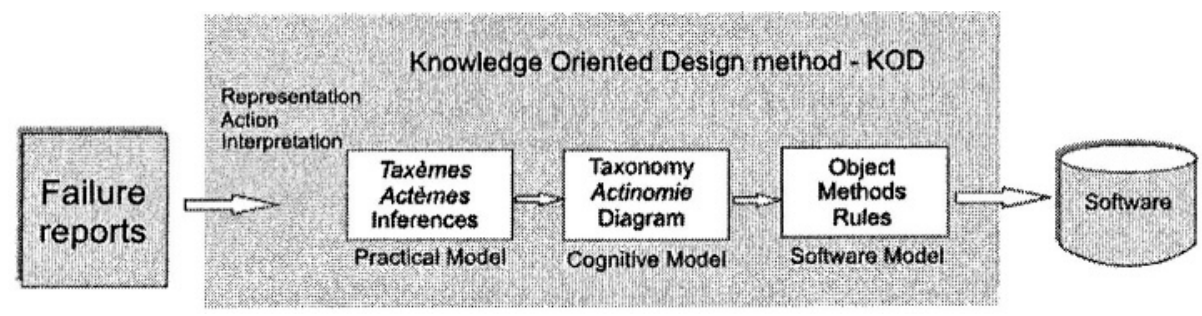

Figure 3. KOD process

The first step consisted in extracting from each raw document, from the corpus, all the elements relevant to the context of study. The terms extracted consisted in all the objects used for the task completion. Each one of those elements had to be described in a later step. The documents consisted of incident reports as well as documents from ANEEL, the Brazilian Electricity Regulatory Agency, which establishes the operator's tasks and the necessary tools and equipments.

The following step consisted in listing all the activities and objects mentioned in each document analysed, according to the action and representation aspects of the KOD paradigm. Elements from interpretation aspect of the KOD paradigm were not available on the reports analysed.

\subsection{Practical Model}

The practical model represents the discourse by means of the elements which belong to the domain of the problem to be solved. In this phase the aim is to formalise the elements extracted from the text and represent the discourse originally expressed in natural language by means of taxèmes, actèmes and inferences. 


\subsubsection{The taxèmes}

The taxèmes are items extracted from the reports, which define the physical and conceptual objects used by experts in the domain. A list with 44 taxèmes was built. These have been formalised in triplets <object, attribute, value $>$. The taxèmes characterise an object from the real world, manipulated by the expert, performed by means of a relation (attribute) which links the object to a value. There are five types of relations: classifying (is-a, type-of, ...), identifying (is), descriptive (position, failure mode,...), structural (composed-of) and situational (is-in, is-below, is-above,...). To illustrate the taxemes, the list below refers to a type of object from the class - switch.

\section{Taxemes for the switch 101-cp:}

<Switch, type of, Interaction <Switch 101-cp, composed of, Device >

green lamp >

<Switch 101-cp, type of, <Switch 101-cp, composed of,

Switch> 1d-label>

<Switch 101-cp, composed of, <Switch 101-cp, composed of, red lamp > 1d-label>

<Switch 101-Cp, composed of, <Switch 101-cp, composed of, 1d-1abel>

handle >

< Switch Sw13-cp, is a, Switch 101>

< Switch Sw15-cp, is a, Switch 101>

< Switch Sw14-cp, is a, Switch 101>

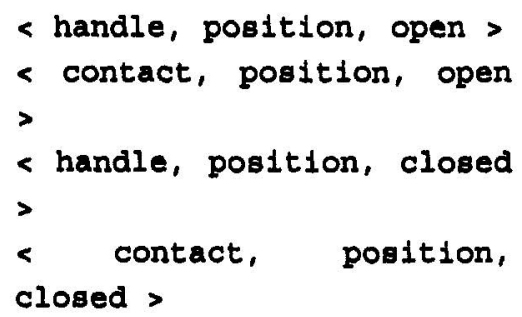




\subsubsection{The actèmes}

The actèmes are textual items extracted from reports, which describe the change of state of an object or concept used by the domain experts. In this case study a list with 52 actèmes has been built. Once identified, the actèmes are translated into a 7-tuple: <Action Manager, Action, Addressee, Properties, State 1, State2, Instruments $>$

- An Action Manager who performs the action

- An Action which causes the change

- An Addressee who undergoes the action

- The properties represent the way the action was executed

- The State 1-is the state of the addressee before the change

- The State $2-$ is the state of the addressee after the change

- Instruments - one or a set of instruments used to cause the change.

Figure 4 illustrates one of the actèmes of the model - 'To Close'.
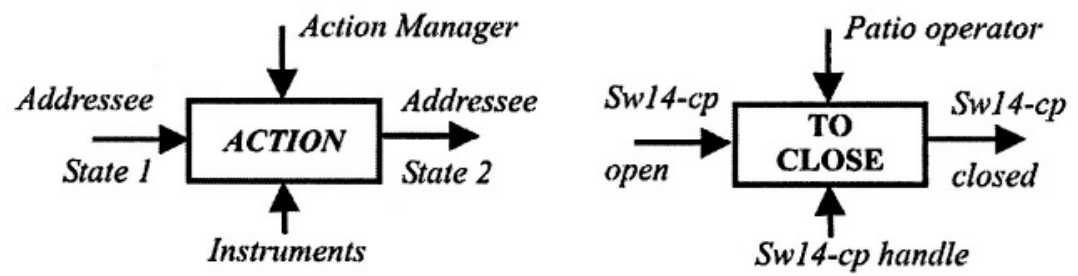

Figure 4. Representation of the Actème 'TO CLOSE'

Table 1. Actème 'TO CLOSE'

\begin{tabular}{lc}
\hline & TO CLOSE (action on the switch 101) \\
\hline $\begin{array}{l}\text { Components } \\
\text { Action Manager }\end{array}$ & $\begin{array}{l}\text { Values } \\
\text { [control room opera } \\
\text { regional centre oper } \\
\text { [Switch 101 of SW }\end{array}$ \\
Addressee & SW14-cp; Switch 101 \\
State 1 (addressee) & 1) [closed ; opened] \\
1) handle-position & 2) [closed ; opened] \\
2) contact-position & 3) [on, off, burnt] \\
3) Red-Lamp state & 4) [on, off, burnt] \\
4) Green-Lamp state &
\end{tabular}

State 2 (addressee)

1) handle-position 1) [closed ; opened]

$\begin{array}{ll}\text { 2) contact-position } & \text { 2) [closed ; opened] }\end{array}$

$\begin{array}{ll}\text { 3) Red-Lamp state } & 3 \text { ) [on, off, burnt] }\end{array}$

$\begin{array}{ll}\text { 4) Green-Lamp state } & \text { 4) [on, off, burnt] }\end{array}$ 


\section{TO CLOSE (action on the switch 101)}

Instruments

[operator's hands, electrical command]

Properties

Unknown (not mentioned in analyzed reports)

The next step, after building a practical model for each report, consisted on building the incident conceptual model or cognitive model, which will be presented in the following section.

\subsection{Cognitive Model}

The cognitive model is the abstraction of the practical models. It is composed of: Taxonomies, Actinomies and Interpretation schemas. The taxonomies are the result of the taxéme classification. It is presented as a tree structure showing the connections between each concept and related objects, as illustrated in Figure 5.

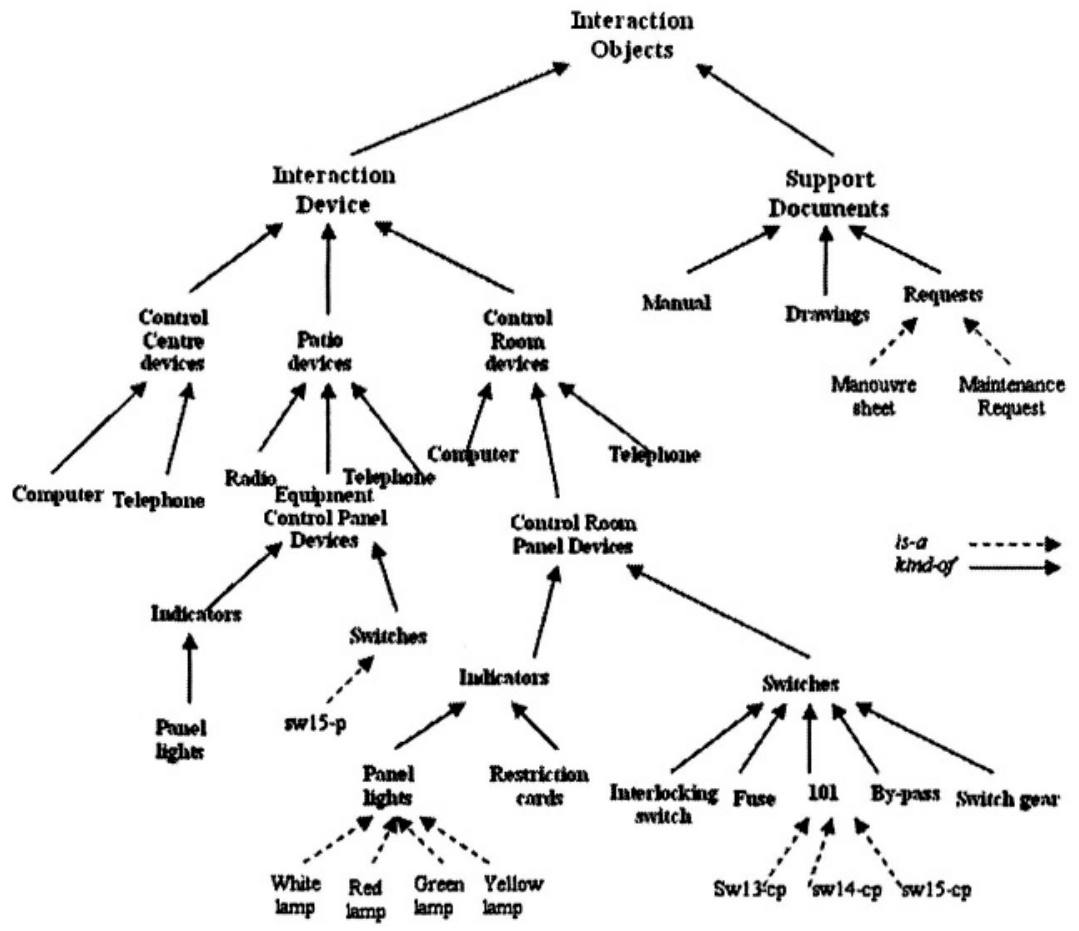

Figure 5. Example of Taxonomy 
Each concept (represented in bold) must be defined, as exemplified in Tables 2 and 3. Switch is an interaction object used by the operator to select one amongst the possible states of a system element. A switch might also be associated to indicators which allow the operator to identify its current state.

Table 2. Switch Concept

\section{Name: Switch}

Reference:

Composed of: \{handle, lamp, red lamp, green lamp, contact, id-label\}

Located: \{on equipment control panel, on control room panel \}

Failure_mode: \{broken handle, lamp always on, lamp always off, lamp burnt, contact always opened, contact always closed $\}$

Table 3. Switch-101 concept

Name : Switch 101-cp

Reference: SW13-cp, SW14-cp, SW15-cp

Composed of: \{handle, red-lamp, green-lamp, id-label, contact $\}$

Localisation : on control room panel

Failure_mode: \{broken handle, red-lamp always on, red-lamp always off, green-lamp always on, green-lamp always off, red-lamp burnt, green-lamp burnt, contact always opened, contact always closed \}

The actinomies are the result of the actémes organisation according to scenarios of human errors. It follows the textual description of an incident scenario taken from one report of corpus analysed in the case study:

'During a manoeuvre to release the switch SWI5-p, it ignored the electrical command for opening, nor remote, or local. So, the patio operator, performing the manoeuvres on the switches, moved to the control room, and there arriving, received a request from the control room operator to agree the position of the switch SW15-cp with that of the red lamp (closed) and open it after, since he was at the telephone with the region's control operator agreeing on other manoeuvres. The patio operator moved to the control panel and unduly moved the handle of switch SW13-cp opening it. Noticing the error closed it after. Soon afterwards he moved the handle of switch SW14-cp opening it, interrupting the load of 1.7 MW, noticing his second error closed the switch. Continuing, he moved the handle of switch SW15-cp in order to open it, but it did not respond to his action remaining 
closed. Finally, he communicated his colleague (control room operator) all his errors and problems with the switches.'

It follows the actèmes used to represent this particular scenario:

- To open <operator, to open, switch, property, switch closed, switch open, switch handle, hands $>$

- To close <operator, to open, switch, property, switch open, switch closed, switch handle $>$

- To move <operator, to move, operator, property, place of departure, place of arrival, feet $>$

- To request <operator1, request, operator2, property, operator2 without request, operator 2 with request, communication tool $>$

- To communicate <operator1, communicate, operator2, property, operator uninformed, operator informed, communication tool) $>$

- To identify a problem<operator, to identify a problem, switch, property, unidentified problem, identified problem, cognitive system>

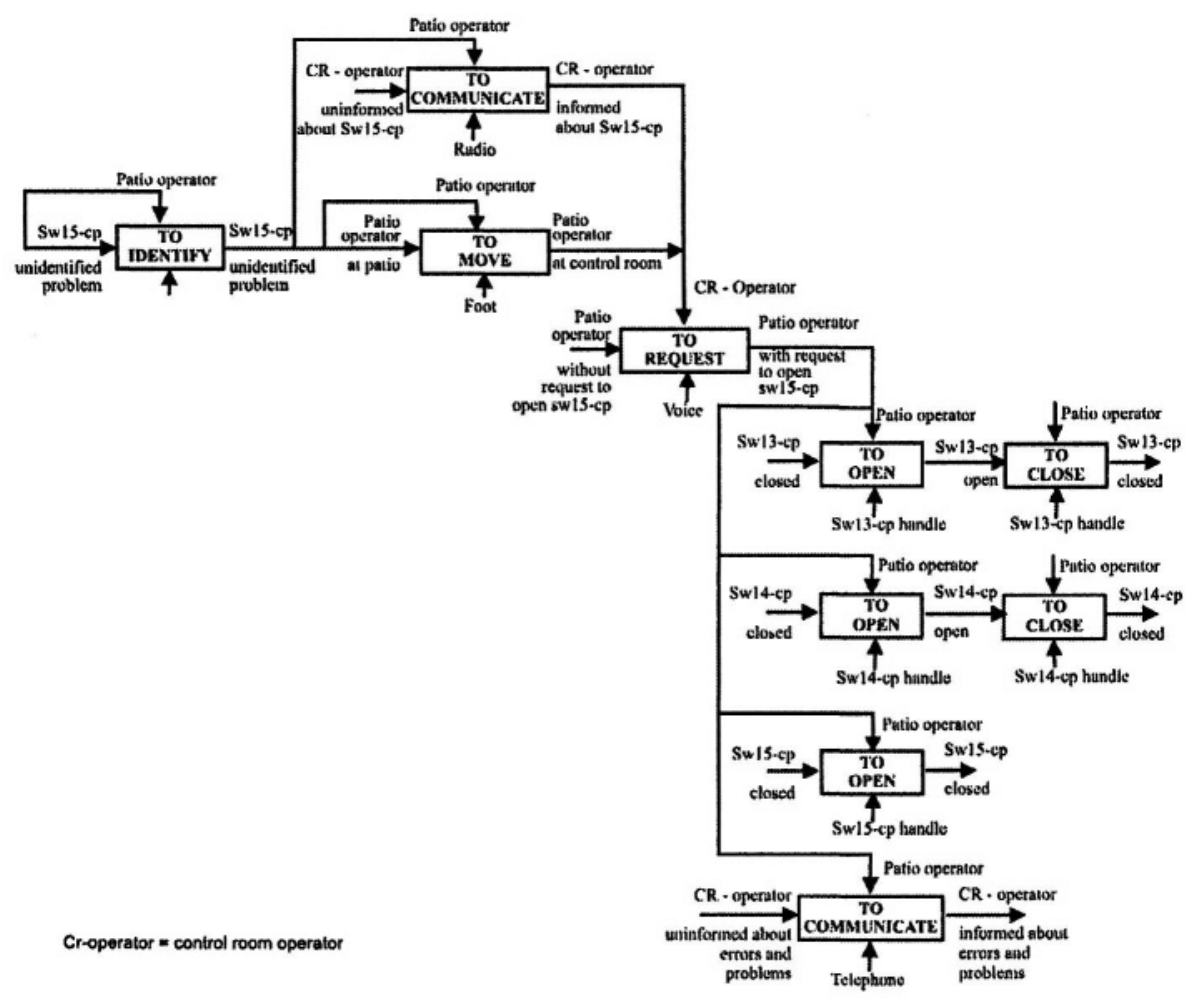

Figure 6. Example of actinomie for the case study scenario 


\subsection{Software Model}

The software model is the formalisation of the conceptual model expressed in a formal language, independent of any programming language or computer platform. It must be expressed in high level and adapted to the nature and complexity of concepts to be represented. It consists on the integration of the taxonomy and concepts into the definition of classes and objects to be used in the development of the software. This model will only be built after completing this preliminary study with the analysis of a more significant number of reports.

\section{CONCLUSIONS AND FUTURE DIRECTIONS}

The use of KOD in this new domain has proven to be adequate to the extraction and organisation of the knowledge required to continue our research work.

One difficult faced consisted in understanding the documents composing the reports, since the industry's objective when writing them was to register the incident causes in order to access responsibilities and propose new work practices. Thus, some of the information there mentioned was not relevant from our research point of view whereas the relevant information was not always clear or complete. So, during the process of knowledge extraction the assistance of the technical personnel from the industry was crucial to understand the description of the systems and tools used to perform the tasks, the context in which the incidents happened, the description of environment where they occurred and particularly the terminology used in the reports (jargon, abbreviations,...).

Once the knowledge extraction is completed, one intended contribution from our work to the industry will consist in proposing the inclusion of aspects related to the operator's cognitive load and the explanation of how the experts (psychologists and engineers) who analyse the causes of incident arrive to the conclusions which are stated on the reports.

It is yet too soon to draw conclusion based upon the sample of reports used in this preliminary study. Thus it is evident the need for a wider analysis of the incident data base in order to represent the incident scenarios more precisely and thus build a simulator capable of better representing the reality of the industry. The model presented in the previous section represents in a structured way the essential knowledge for building the incident simulator, since it provides information on the scenarios and on the objects and actions performed by the operator during task execution. 
On the other hand, although the corpus analysed is still very modest compared to number of cases available in the industry, it already allows us to identify the critical aspects of the operator's interfaces with the system, giving insights on how to improve their design in order to avoid the reappearance of problems. This is in itself a contribution to the design of interfaces for critical systems in the studied domain. However, a cognitive model of the operator's behaviour when dealing with incidents is still needed in order to understand the reasons which lead the user into carrying out wrong actions. This understanding will allow us to conceive systems which empower the user avoiding the occurrence of these errors.

Once this model is available the next step of this research project will consist in building the simulator and defining an experimental protocol for the tests which will be initially performed in the controlled environment of the lab, and later in the real work environment of the industry. After being validated, this model will be used in the conception process of user interfaces for critical applications, using the method MCIE. This approach of user interface development is expected to make them more adequate to the user cognitive needs and thus result in a reduction on the number of errors related to the interaction between operators and safety critical systems.

\section{ACKNOWLEDGEMENTS}

The authors would like to thank the agencies CAPES and COFECUB for partially financing this project.

\section{REFERENCES}

1. Amalberti, R. La conduite de systèmes à risques. Collection Le Travail Humain. 1996

2. Ganascia, J. G. Securité et Cognition. Editions HERMES. 1999

3. Guillermain, H. Ferrer, P. S. Contribution à l'identification des risques facteurs humains dans la conduite des processus à haut niveau de sûreté de fonctionnement, Securité et Cognition. Editions HERMES. 1999

4. Vogel, C. Le genie cognitif. Masson. 1988.

5. Hollnagel E. Cognitive Reliability and Error Analysis Method CREAM. Elsevier. 1998

6. Turnell, M. F. Q. V., Scaico, A., Sousa, M. R. F.; Perkusich, A. "Industrial User Interface Evaluation Based On Coloured Petri Nets Modelling and Analysis". Lecture Notes in Computer Science - Interactive Systems, LNCS 2220, p. 69-87, Germany, 2001.

7. Hammouche, H. De la modélisation des tâches utilisateurs au prototype de l'interface homme-machine, Thèse de Docteur, Université Paris VI, Décembre, 1995.

8. Furtado, M. E. S. Mise en oeuvre d'une méthode de conception d'interfaces adaptatives pour des systèmes de supervision à partir de spècifications conceptualles, $\mathrm{PhD}$ thesis, Doctorat de productique et Informatique à l’Univcrsité Aix Marseille III, France, 1997. 
9. Gamboa, F. R. Spécification et Implémentation d'ALACIE: Atelier Logiciel d'Aide à la Conception d'Interfaces Ergonomiques, Thèse de Doctorat, Paris XI, Octobre, 1998.

10. Guerrero, C. V. S., Lula, B. Jr. Model-Guided and Task-Based Approach to UI Design Centered in a Unified Interaction and Architectural Model, CADUI'2002 - 4th International Conference on Computer-Aided Design of User Interfaces, Valenciennes, FRANCE, May 2002

11.Turnell, M.F.Q.V., Farias, G.F. The use of Supervisory Software in the Industrial Automation Process Control from the User Interface Perspective. 1996 IEEE International Conference on Systems, Man and Cybernetics; Beijing China, October, 1996.

12.Sousa, M. R. F., Turnell, M. F. Q. V. "User Interface Design Based on Coloured Petri Nets Modelling and Analysis". Proceedings of the 1998 IEEE International Conference on Systems Man and Cybernetics, San Diego, USA, 1998. S 2220, p. 69-87,

13.Mercantini, J.M., Capus, L., Chouraqui, E., Touriny, N., Knowledge Engineering contributions in traffic road accident analysis. In Innovations in Knowledge Engineering, pp 211-244, Ed. Ravi K. Jain, Ajith Abraham, Collette Faucher, Berend Jan Van der Zwaag. 2003.

14.Mercantini, J.M., Loschmann, R., Chouraqui, E. A provisional analysis method on safety of an urban industrial site, In Safety in the Modern Society, People and Work Research Report 33, pp 105-109, ISBN 951-802-338-7, 2000. 